\title{
Association of Social Network Analysis with Mental Models in Emergency Management Teams
}

Bjørn Sætrevik \& Line Solheim Kvamme

Operational Psychology Research Group, Faculty of Psychology, University of Bergen, Christies gate 12, NO-5015 Bergen.

\begin{abstract}
Social network analysis is a preferred approach to examine the impact of social processes and mechanisms on team performance, but it can be challenging to measure these dynamics in applied settings. Our aim was to test whether the understanding of the task at hand was more accurate and more shared for teams with more evenly distributed interaction patterns. We pre-registered a novel approach for measuring social networks from sparse reporting of ranked interactions. Our sample was eleven emergency management teams that performed a scenario training exercise, where we asked factual questions about the ongoing task during performance, and retrospective questions about who were the most important communication and collaboration partners. We quantified shared mental models as the extent to which a team member showed the same understanding as the rest of their team, and quantified situation awareness as the extent to which team members showed the same knowledge as their team leader. We calculated which team members where most central to the network, and which networks had more evenly distributed networks. Our findings support the pre-registered hypotheses that more interconnected teams are associated with more accurate and more shared mental models, while the individual's position in the network was not associated with MM.
\end{abstract}

\section{Keywords}

Social network analysis; mental models; shared mental models; situation awareness; emergency management teams; teamwork; social interaction

\section{1: Introduction}

\section{1: Theoretical background}

1.1.1: The role of social networks in team performance. Most work is performed by some form of team (Cross, Rebele, \& Grant, 2016). Teamwork requires coordination between team members, sharing of responsibilities, assigning team roles and using explicit communication (Salas, Sims, \& Burke, 2005). There are advantages to working in teams, such as being able to solve more complex tasks, specialization, mutual supervision, and collaboration across domains. But it also carries potential disadvantages, like coordination costs, conflicting goals, overlapping resource use, miscommunication and groupthink. A team's formal and informal organization may be considered its social structure, while procedural, instrumental, and affective interactions may be considered its social processes. Certain team structures and social processes may promote the advantages of teamwork while minimizing the disadvantages (see for instance, Bolstad \& Endsley, 2005; Cummings \& Cross, 2003; De Jong, Dirks, \& Gillespie, 2016). Therefore, it may be beneficial to analyze a team's social dynamics to learn what types of patterns optimize the team's performance.

Emergency management teams (EMTs) operate in dynamic situations, where the stakes are high, and decisions are continuously made under time pressure. Information and feedback tend to be sparse and unreliable, and there are limited opportunities for communication and coordination. To attain optimal team functioning in such settings the team members must communicate closely and be able to rely on each other. Our aim was to explore whether the EMT's social processes and structures influence their performance, in terms of having a shared understanding of the task and their work.

Social network analysis (SNA) explores the interaction between interconnected agents by graphing dyadic relationships between relevant individuals, groups or institutions (Borgatti \& Foster, 2003; Henttonen, 2010). The approach assumes that individuals are embedded in multilevel relational configurations (Balkundi \& Harrison, 2006; Monge \& Contractor, 2003; Newman, 2010; Streeter \&
Gillespie, 1993) where the social network has structure (nodes and ties) and content (frequency and importance of the ties, distribution of resources). Social network structures may be described in terms of their popularity, which indicates the number and significance of ties a node receives (Opsahl, Agneessens, \& Skvoretz, 2010), point centrality, which indicates the degree to which a node is associated with other nodes (Sparrowe, Liden, \& Kraimer, 2001), centralization, which indicates the degree to which all nodes are equally central (Cummings \& Cross, 2003), and density, which indicates the extent to which a network's nodes are interconnected (Hanneman \& Riddle, 2005). SNA is typically applied to rich, longitudinal data, making it ideal for uncovering patterns in large social structures where interactions may be automatically recorded, such as in examining frequency of email correspondence or development of connections in social media (Borgatti, Mehra, Brass, \& Labianca, 2009).

Previous research has indicated that teams with more distributed social network profiles are more effective for team performance (Balkundi \& Harrison, 2006), and that denser and more distributed EMT networks may reflect team cohesion and coordination (Mohammadfam, Bastani, Esaghi, Golmohamadi, \& Saee, 2015). Network density and distribution has been shown to be related to team performance, but results are ambiguous and sometimes contradictory (Kilduff \& Tsai, 2003). A number of studies (Cummings \& Cross, 2003; Henttonen, 2010; Leavitt, 1951; Leenders, van Engelen, \& Kratzer, 2003; Lin, Yang, Arya, Huang, \& Li, 2005; Mehra, Dixon, Brass, \& Robertson, 2006; Shaw, 1954, 1964; Sparrowe et al., 2001) have shown higher performance and efficiency on complex tasks for more distributed teams (i.e. where centrality is equally distributed, as opposed to focused around one or a few nodes). A metaanalysis (D’Innocenzo, Mathieu, \& Kukenberger, 2016) found lower centralization and increased density to be associated with increased team performance in 50 effect sizes across over 3.000 team members. The trend across different SNA indices appears to be that more decentralized teams are 
more effective, while it appears to be more difficult to predict team member performance from SNA.

A positive association of network distribution on team performance may be because network's interaction patterns determine the flow of information in the team (Cross, Borgatti, \& Parker, 2002; Haythornthwaite, 1996). Some networks may allow most members to calibrate their beliefs with each other, while others have central members that function as bottlenecks for the information flow. It has been argued (D'Innocenzo et al., 2016) that distribution and density in team networks are indicators of the leadership role being shared across team members. "Shared leadership" may lead to more autonomy and competence by causing team members to take on more responsibility and make more autonomous decisions, maintain close collaboration with the rest of the team, bring more resources to the team, share more information, and experience higher commitment. As EMTs are typically small and have highly specialized team roles, their degree of distribution may reflect a mechanism related to shared leadership.

1.1.2: Mental models in team performance. Team performance may be difficult to assess in operative settings, as tasks tends to be complex, dynamic and oblique (Carayon, 2006; Osman, 2010), and even structured training scenarios may play out in idiosyncratic ways that makes it difficult to set normative criteria for evaluation. The understanding, or mental models (MM) that the team members have of their task and their work may be an indirect indicator of performance, as it may be expected to determine the quality of decision being made (Bolstad \& Endsley, 1999; Endsley, 1995). The ability for team members to build MM is determined by the extent to which the team's interaction network provides them with access to information.

The concept of shared mental models (SMM; CannonBowers, Salas, \& Converse, 1993) represent the extent to which team members have the same understanding of their collective work. SMM can be related to the team's knowledge about their equipment, their task, their interaction, and team member capabilities. The extent to which a team has SMM has been found to correlate with team performance (see for instance Mathieu, Heffner, Goodwin, Salas, \& CannonBowers, 2000; Rouse, Cannon-Bowers, \& Salas, 1992; Santos, Uitdewilligen, \& Passos, 2015; Stout, Cannon-Bowers, Salas, \& Milanovich, 1999). SMM may be particularly important in high-stakes settings, where limitations in information, resources and time, may be alleviated by having an updated and shared understanding of the ongoing situation. Some aspects of SMM overlaps with the concept om transactive memory systems (Lewis \& Herndon, 2011; Zhang, Hempel, Han, \& Tjosvold, 2007).

While the degree to which all members have similar MMs may be an indicator for the team's coordination, the quality of the team member MMs may be indicated by the accuracy of their situation awareness (SA) in representing relevant aspects of the task situation. Endsley (1988, p. 97) defined SA as "the perception of the elements in the environment within a volume of time and space, the comprehension of their meaning and the projection of their status in the near future". It is worth noting that there are discussions around the conceptualization and measurement of SA (see Sarter \& Woods, 1991; Stanton, Salmon, Walker,
Salas, \& Hancock, 2017). For the current purpose, we will measure SMM as the extent to which all team members have the same understanding, while we will measure SA as the extent to which a team member's understanding matches the understanding of the best-informed team member.

\section{2: Research needs}

1.2.1: Indices for social network in impoverished settings. It has been argued that SNA should be a preferred approach to explore team performance (Borgatti \& Foster, 2003; Burt, Kilduff, \& Tasselli, 2013), and that the approach is currently underused (Balkundi \& Harrison, 2006; Henttonen, 2010; Wölfer, Faber, Hewstone, \& Marcus, 2015). SNA research is well suited for investigating complex data where nodes and ties in a community have been recorded over an extended period of time. However, it is unclear how SNA should be applied in cases where a small team works on a shared task for a limited period, and ecological concerns prevent direct measurement of the social processes. Having to resort to retrospective selfreport in a busy setting may lead to missing or mis-recorded data, and few data-points from which to quantify the network. Thus, there appears to be a need for suggesting and testing methodologies and indices that can quantify social networks in ecological field settings, and that can be applied to settings such as EMT work. Thus, one aim of the current study was to test the feasibility and suitability of some suggested measures and analysis approaches for SNA and MM in impoverished field datasets.

1.2.2: Social networks and mental models. As discussed above, network properties may be expected to predict team performance. One may assume that properties of the social network in teams working under challenging circumstances will impact the team's ability to form accurate MMs. However, few previous studies have used SNA during taskwork in operative settings to explore social dynamics or test their associations with MMs. Previous research does not inform us about which SNA indicators (i.e. centrality or centralization) would predict team performance in the setting such as EMTs, and whether effects would be associated with MM measures (shared MM or SA). Thus, a second aim of the current study, was to test whether any of our current SNA measures were associated with MM measures. This may provide guidance to how the measures can be used in further research and in training.

\section{3: Current study}

1.3.1: Research setting. The current research was conducted on the entire emergency management organization of a large offshore hydrocarbon energy company. The organization has six second line EMTs, each consisting of nine roles with defined tasks and responsibilities. Upon notification of emergency incidents on offshore installations the team musters in a dedicated room. The team collects information about the incident, predicts its development, advises first and third line emergency management, distributes resources to handle the emergency, and alerts other parties. The team's leader (the chief of staff) assigns tasks, develops plans, and initiates status update meetings. The teams were asked to perform two realistic training scenarios for a total of eleven sessions. 
1.3.2: Hypotheses and preregistration. Building from the assumption that the individual team members' position in the network can impact their MM, we pre-registered the following hypotheses: Individual team members' centrality (reflecting higher popularity) in the communication network will be positively associated with individual SMM (H1a), as will centrality in the reliance network (H1b). Further, individual team members' centrality in the communication network will be positively associated with individual SA (H1c), as will centrality in the reliance network (H1d).

Building from the assumption that the teams' overall network centralization has a negative effect on MM, we preregistered the following hypotheses: Members of teams with less centralized (i.e. more distributed) communication networks will be associated with team SMM (H2a), and similarly for teams with more centralized reliance networks (H2b). Further, members of teams with less centralized communication networks will be associated with team SA $(\mathrm{H} 2 \mathrm{c})$, and similarly for teams with more centralized reliance networks (H2d).

The pre-registration and related files are available on the Open Science Framework (OSF): https://osf.io/uvsta. At the time of pre-registration, the dataset had been collected, individual and team's MM scores had been calculated, and their relationship to changes over time and to team membership had been analyzed (Sætrevik, 2015; Sætrevik \& Eid, 2014), but SNA indices had not been compiled, and associations between SNA and MMs had not been tested. ${ }^{i}$ Due to theoretical and methodological development during the project, there were some deviations from the pre-analysis analysis plan, as described below.

\section{2: Methods}

\section{1: Measurement}

2.1.1: Procedure. We performed the analyses on an extant dataset of eleven second line EMTs. Data was collected during 2-3 hour long training scenario exercises in an emergency room, where the regular teams used the equipment that would be involved in an actual event. The scenario was to respond to either a fire and personnel injury (sessions 1-6), or a blow-out incident (sessions 7-11) on an offshore oil producing rig. The research team was involved in planning the training scenarios to suit the purposes of the study. One or two researchers were present at every exercise to explain the purpose of the study and answer pertinent questions, to distribute materials and to call attention to answering them during the freeze points. MMs were measured during freeze points at regular intervals during the task work, while SNA was measured at the end of the exercise. There were minor differences in study design between the first six and the last five sessions, please see details in a previous publication (Sætrevik, 2015).

2.1.2: Social network measurement. At the end of each scenario exercise all team members answered questions about who they had communicated most, second most, and third most with. Immediately following, they answered questions about who they had relied on most, second most, and third most to complete their tasks in the scenario. For each question, they responded by selecting the name of one of the team member roles from a list. Upon examining the responses from data collection, we found that some responses were made incorrectly, and we registered a plan for how to handle this (see OSF project folder).

We calculated each team member's centrality in the network by counting their number of incoming ties each team member had, and in order to retain data depth weighting the ties with a factor of 3,2 or 1 according to whether the interaction was marked as "most", "second most" or "third most" important. The weighted popularity was dividing by the team's number of responses, to avoid being confounded be missing responses. This results in a weighted popularity score between 0 (if no one in the team indicated that they had interacted with you) and 1 (if you were the only one in the team that the others had interacted with).

In order to attain a measure of each team's overall degree of centralization, we calculated the sum of squared differences of the team's weighted popularity scores. This yields a representation of the extent to which the team as a whole report a few team members that they interacted most with (a score of 1 if the whole team only interacts with a single team member), as opposed to a more distributed interaction (a score approaching 0 if all team members interacts equally much with each other).ii Approaches such as density calculation would not be applicable to the current dataset, as the materials requests three responses from all members, and variation in density would only represent the extent to which responses are missing or ties are bidirectional, which would be difficult to interpret.

2.1.3: Mental model measurement. Participants' MMs were measured by questions at freeze points throughout the scenario. At each freeze point all team members were asked to answer probe questions in a paper booklet (session 1-6) or in an online questionnaire (session 7-11). The first question asked where the incident was active (chose from list of four different platform sections), the second asked which type of incident was ongoing (chose from list of twelve different incident types), the third asked how many of the personnel were missing, injured and deceased (write down three numbers), the fourth asked what the consequences of the incident would be (indicate the likelihood of seven different possible outcomes), and the final probe asked the team's current priorities were (select what was most, second most and third most important to work on from a list of 12 different courses of actions). More details can be found elsewhere (Sætrevik, 2012), or in the experiment materials online (https:/ / osf.io/uvsta).

The first author's previous research on the current dataset (Sætrevik, 2015; Sætrevik \& Eid, 2014) has suggested the following measurement approaches for MMs in the current setting. SMM was indexed by calculating the extent to which each team member answered the freeze point questions in the same way as the rest of the team. Previous work has also suggested that SA could be indexed by the extent to which team members agree with the team leader's answer to the freeze point questions. As the team leaders' function involves gathering and distributing information within teams, it is reasonable to assume that they are the best-informed members of the team. The team leader's assumptions about the state of the task were thus used to assess the accuracy of the remaining team members' SA. Some limitations to this 
approach in discussed in previous publications, such as the leader not necessarily always being better informed or objectively correct. Both measures yield scores from 0 to 1 , ranging from no overlap to complete overlap between the team member's responses and the team's average or the team leader's responses. SMM and SA scores for each team member were averaged across the freeze points for the analyses. Full datasets showing stepwise calculation of indices are available on the OSF project folder, as well as a spreadsheet that demonstrates the calculations.

\section{2: Analyses}

The current analyses were based on data from eleven teams in two different training scenarios. The composition of the teams varied somewhat between and within the scenarios. For the teams to be comparable, one or two participants were removed from some of the teams so that all teams consisted of only the nine team roles that were present in all teams (total $\mathrm{n}=99$ ). The roles that were included in the analyses were Chief of Staff, Line Leader, Government Liaison, Communications Officer, Personnel Coordinator, Medic, Air Coordinator, Marine Coordinator, and Marine Communication. Two people held the same team roles in three of the teams. In these teams, only the responses from team members acting as the main responsible for the function were retained. In cases where responses from this person were missing, the back-up's responses were used instead.iii

To test the relationship between the team member's position in network and MMs, four simple linear regressions corresponding to hypotheses H1a-d were performed. Similarly, four simple linear regressions corresponding to hypotheses H2a-d were performed to test the relationship between the teams' degree of distribution and the team members' MM scores. In accordance with the preregistration, the inference criteria for these analyses were set at $\mathrm{p}=.05$, one-tailed in the direction indicated by the hypotheses. Transformations and index calculations were done in Google Sheets that are available in the OSF folder. Hypothesis testing was done in RStudio (RStudio Team, 2018). Visualization was done in $\mathrm{R}$ using the iGraph package (Csardi \& Nepusz, 2006).

\section{3: Results}

3.1: Description of social network distribution

Overall, the EMT networks displayed some variance in degree of centralization, as shown for the communication networks in Figure 1. Eight of the eleven teams were clearly centralized around the team leader (Chief of Staff), for both communication and reliance networks. Team B's network was less centralized around the team leader, whereas team E and $\mathrm{F}$ were centralized around a different team member. The team leader had significantly higher popularity scores than the other team members, in both communication networks $(t(97)$ $=5.19, p<.001)$, and reliance networks $(t(97)=8.22, p<$ .001). The Line Leader was also a central member, and was the most popular member for the teams where the team leader was not most popular. This may reflect the Line Leader's function as the second-best informed team member that may in some cases take over some of the leadership functions of the Chief of Staff.
Few differences between the communication and reliance networks emerge by visual inspection. The networks displayed clusters of ties that were aligned with the functions of the team roles. The Chief of Staff, Line Leader, Communications Officer and Government Liaison constituted a "management cluster" in most of the teams (shown in yellow in the figure), Personnel Coordinator, Medic and Air Coordinator constituted a "personnel cluster" (shown in red), while Marine Communications and Marine Coordinator constituted a "maritime cluster" (shown in blue). The communication and reliance networks aggregated across teams show the management cluster in the center, while personnel and maritime clusters are peripheral. There were no direct ties between the personnel and maritime functions in the aggregated networks, which likely reflects that the managerial functions constitute a mediating role between these clusters. Eight of the communication networks had a single tie from maritime to personnel clusters, while two of them also had reciprocal ties. Nine of the reliance networks had ties from maritime to personnel clusters, and three of them also had reciprocal ties.

\begin{tabular}{|l|c|c|c|c|c|}
\hline & Com. pop. & Rel. pop. & Com. centr. & Rel. centr. & SMM \\
\hline $\begin{array}{l}\text { Reliance } \\
\text { popularity }\end{array}$ & .819 & & & & \\
\hline $\begin{array}{l}\text { Communication } \\
\text { centralization }\end{array}$ & $<.000 *$ & $<.000$ & & & \\
\hline $\begin{array}{l}\text { Reliance } \\
\text { centralization }\end{array}$ & $<.000 *$ & $<.000$ & .145 & & \\
\hline $\begin{array}{l}\text { Shared mental } \\
\text { models }\end{array}$ & .059 & .041 & $-.13 *$ & $-.305 *$ & \\
\hline $\begin{array}{l}\text { Situation } \\
\text { awareness }\end{array}$ & .050 & .058 & .025 & $-.206 *$ & .771 \\
\hline
\end{tabular}

Table 1. Matrix showing Pearson's correlation between social network and mental model indices (asterisk indicates significant correlations).

\section{2: Effects of team-members' popularity in the network}

The correlation matrix of the SNA and MM variables are shown in in Table 1 (note that centralization values do not differ between members of a team). The four H1 hypotheses describing associations between each individual's position (popularity) in the networks and MMs were tested with four independent simple linear regressions. None of these tests showed significant associations between individual SNA indices for communication popularity and reliance popularity and $\mathrm{MM}$ measures of SMM and SA (two-tailed p-values = $\left..562-.684, \mathrm{r}^{2}=.002-.003\right)$.

\section{3: Effects of teams' overall network centralization}

Each team's degree of centralization was calculated as the variation of the team's popularity scores, and the association to SMM and SA was tested with four independent simple linear regressions. There were significant negative associations between the teams' reliance centralization and both SMM (H2b: F(2,97) 9.98, $\left.\mathrm{p}=.001, \mathrm{R}^{2}=0.093\right)$, and SA (H2d: $\left.\mathrm{F}(2,86) 3.8, \mathrm{p}=.028, \mathrm{R}^{2}=0.042\right)$, while there was no effect of communication centralization on either SMM (H2a: $\mathrm{F}(2,97) 1.66$, two-tailed $\left.\mathrm{p}=.2, \mathrm{R}^{2}=0.017\right)$ or SA (H2c: $\mathrm{F}(2,86) 0.06$, two-tailed $\left.\mathrm{p}=.815, \mathrm{R}^{2}=0.001\right)$. There were thus effects of teams with more distributed reliance networks having more shared and accurate mental models, accounting for $9 \%$ and $4 \%$ of the variation respectively. 
Team A

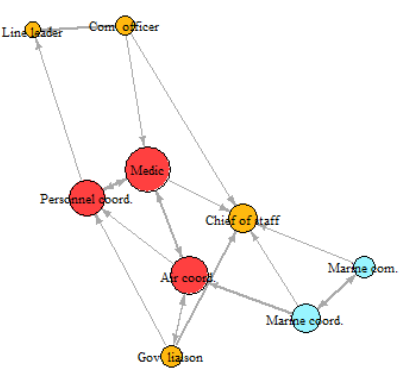

Team E

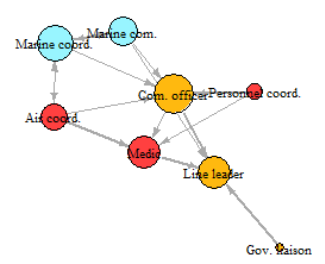

Team I

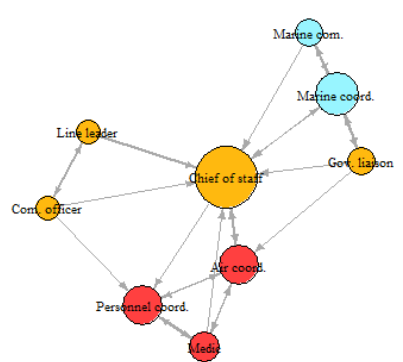

Team B

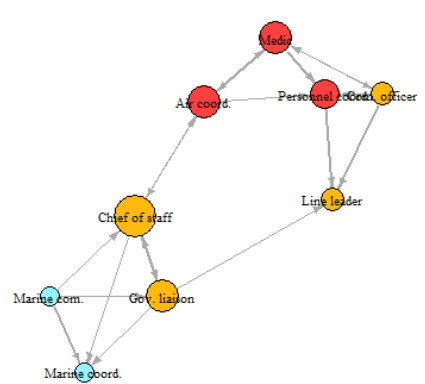

Team F

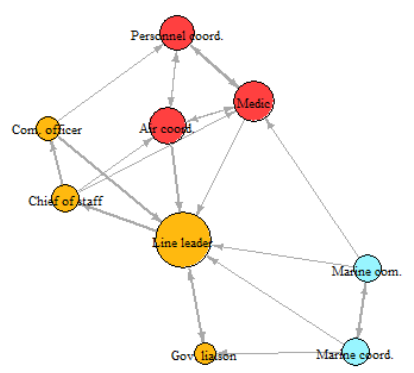

Team

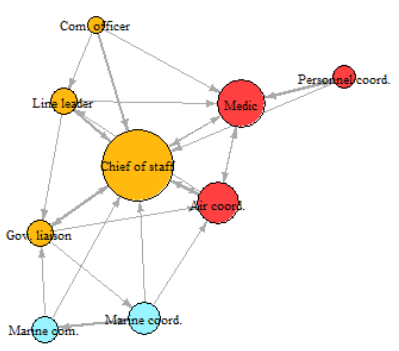

Team C

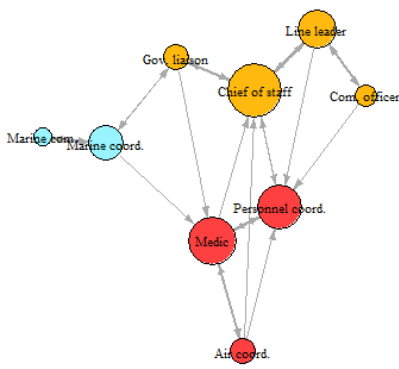

Team G
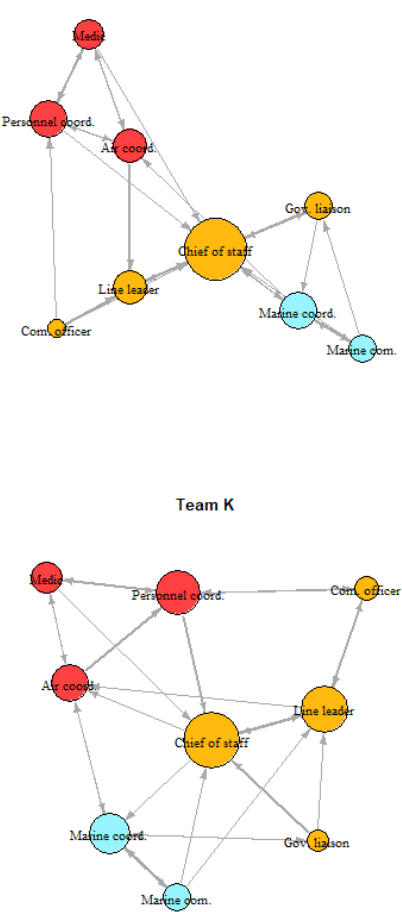

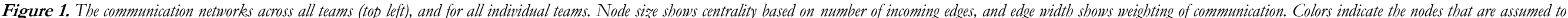
share tasks. Team $G$ is an example of a centralized network, while $A$ is an example of a distributed team. A similar figure for reliance network is available in the OSF project folder. 


\section{4: Discussion}

\section{1: Summary of results}

The current study tested the viability of using SNA in field studies to predict MMs in EMTs. We sought to determine whether patterns of interactions between team members, measured with retrospective self-report, were associated with the similarity of team members' beliefs about the task and their work. We found support for our pre-registered H2 hypotheses, in showing that more distributed team networks of reliance were associated with both more shared MMs and more accurate SA. The H1 hypotheses of individual team member's position in the network being associated with MM quality were not supported. Below we will examine the identified communication and reliance networks, before discussing the implications of the results for the two hypotheses.

\section{2: Communication and reliance networks}

While other SNA studies have looked at networks of "friendship" or "advice", we find that the concepts of "communication" and "reliance" to be more relevant for of a task-focused and time-limited teams and tasks in the current setting, and will be more intuitive for the responders. Our reliance measurement likely coincides with the construct of "trust" between team members, which has previously been argued to be a predictor for team performance (De Jong et al., 2016).

At the end of the scenario exercise we asked team members which three team members they had communicated most with. The communication networks that emerged were mostly centered around the team leader, and had distinctive central clusters comprising of managerial functions, and peripheral clusters of marine and personnel functions, with few ties connecting the peripheral clusters directly, as described in section 3.1. This matches the assumed function and operating modes of the teams, where team members in the management cluster may serve as gatekeepers for the information flow (Cross et al., 2002).

It is worth noting that unlike most SNA research, we did not record interactions directly, but measured team members' recollections and portrayals of the interactions. Since our measure only allowed each team member to describe the three most important ties, there may have been additional ties that the responders were unable to report. However, despite being instructed to report three interactions, on average 2.5 ties were reported. This may indicate that at least some team members did not have much more interaction that they could have reported. We find it to be a reasonable compromise that some of the less important ties may have gone unreported in order to have a concise measure. The questionnaires did not specify that respondents were to report instrumental communication (i.e. relevant to the scenario task). While it is possible that some respondents also reported some taskirrelevant communication given the restrictive task setting this is unlikely to have constituted a major confound. Nevertheless, future studies could specify which type of communication we are interested in to eliminate this possible confound.

At the end of the scenario exercise we also asked team members which three team members they had relied most on to complete their tasks to generate the reliance networks. It has been argued (Costa, Fulmer, \& Anderson, 2018; De Jong et al., 2016; Grossman \& Feitosa, 2018) that mutual trust has prominent effects in teams that are highly interdependent and where there is a clear leader, a description that matches well with the EMTs in our sample. We therefore considered that in addition to communication patterns, the level of reliance in the teams could be a relevant measurement of how interdependency may influence shared beliefs. The reliance networks seen in the current study were characterized by the same clusters as for the communication network (see section 3.1).

A substantial amount of the communication and reliance ties were between members within the maritime and within the personnel clusters. The communication network also had several non-reciprocal ties from maritime cluster to personnel cluster team members. This may illustrate that the maritime functions needed to communicate with personnel, whereas the personnel functions to a larger extent communicated with the managerial functions. The reliance networks had more bidirectional ties between the clusters, in particular between the Marine and Air Coordinator functions. This indicates that team members may see that they are mutually contingent on each other to complete a task, even if the communication goes mainly in only one direction.

\section{3: Network associations and individual performance (H1)}

Tests of our H1 hypotheses did not support an association between team member's mental models and their popularity in the communication or reliance networks. We thus did not find support in this study for the assumption that more centrally positioned team members had better access to information that allowed them to have MMs that were more aligned with the rest of the team and the team leader. This contrasts with previous studies that have shown node popularity to be associated with individual performance measures. The current null-finding for $\mathrm{H} 1$ is also in contrast to the tests of $\mathrm{H} 2$, which showed that properties of the team's network as a whole did influence MM.

The lack of effect of individual team position may be due to the teams in our sample being small, highly interdependent and focused around a shared task. Even though there are distinct roles and a clear leader within the team, the team's ability to form MMs likely depends on smooth operation of team dynamics across positions in the hierarchy or occupational discipline. Further, it could be that (apart from the two team members with leadership roles standing out) there was insufficient variation in popularity among the team members to show effects on MMs. Further, it has recently been suggested (Wise, 2014) that measures of cohesion may have an inverse curvilinear relationship to performance, so that increased cohesion is better up to a point, but thereafter "everybody talking to everyone else" may decrease performance. Such complex relationships may make positive associations difficult to find in sparse datasets.

\section{4: Network associations with team performance $(\mathrm{H} 2)$}

Tests of the $\mathrm{H} 2$ hypotheses supported the assumption that MM quality would be higher for team members in more distributed teams. This indicates that in teams where interactions were more evenly distributed between the members (as opposed to interactions being centralized), team 
members had MMs that were more similar to the rest of the team, and that were more similar to the team leader's MM.

A previous analysis of the current dataset (Sætrevik, 2015) found that EMT members' degree of shared beliefs were affected by team membership. This was attributed to team specific characteristics, such as leadership, shared experiences, or communication and collaboration patterns. The current study attempted to explore some of these possibilities by testing associations between communication and reliance networks with shared beliefs. As a number of previous studies have indicated that more centralized teams are less efficient and score lower on performance outcomes (see e.g. Cummings \& Cross, 2003; Henttonen, 2010; Leenders et al., 2003; Lin et al., 2005; Mehra et al., 2006; Sparrowe et al., 2001, and section 1.1.1.), the degree of centralization in the teams may be one of the factors accounting for the variation in team performance in these teams.

The current measure of team level centralization indicates the extent to which some team members are more popular than others (as opposed to all team members have similar popularity), while taking into account the ranked responses and missing responses. A more distributed team has more possible pathways to transfer resources, such as information, task distribution or support. We believe that using communication and reliance centralization as the variation in a team's weighted popularity as predictors for shared beliefs may be a useful approach for future research in operative teams, as it likely reflects patterns of flow and distribution of information in small teams where interactions are sparse and can be ranked.

Accurate and shared MMs are assumed to facilitate team cooperation and overall team performance (Bolstad \& Endsley, 1999; Mathieu et al., 2000). The current results thus indicate that the centralization of EMT's networks could lead to improved task performance. This could happen through more distributed communication and reliance leading to more efficient dissemination of information between team members. Another mechanism could be that the team leader's role in distributing relevant information to the rest of the team may lead to efficient and task-relevant coordination of reliance and communication. More centralized teams may have leaders or other team members that act as "gatekeepers" who restrict the flow of information, creating an obstructive "bottlenecks" that delays and restrict the team's performance (Balkundi, Barsness, \& Michael, 2009). Although the current teams have a hierarchical organization, extensive training and performs status meetings that structure the task work, our SNA measures indicate that the information pathways vary within our sample and may be associated with facilitating collaboration. Measures such as density and centralization have both been found to be predictive of team outcomes such as "shared leadership" (D'Innocenzo et al., 2016). That the leadership role is shared among team members may contribute more resources, share more information and display more commitment, which has been shown to improve team performance.

\section{5: Implications}

4.5.1: Suggestions for future research. To summarize our findings, the teams' overall network properties were associated with the team members' ability to achieve shared and accurate MMs, while the individual team member's position in the network was not. This suggests that SNA may be a relevant measure for the team processes that predicts MM quality in operative teams. We recommend similar measurements and analytical approach for future research, but some adjustments could be considered. For example, as there was only effects of the reliance networks in the current network, it may be superfluous to measure both communication and reliance. Using only a single network to index team interaction would have made for a more efficient study and analysis. However, as discussed above (section 4.3), the above results showing diverging communication and reliance networks indicates that there may be value in analyzing them separately. For future use in teams where the communication and reliance networks are assumed to coincide, it may be excessive to measure both concepts.

While the current study measured both SMM and SA, it could be argued that the external validity is weaker for the SA measure. Since the SA measure is based on a reference score, it makes an a-priori assumption about who is the bestinformed team member, which may not be applicable for all settings. For teams where it is not given which individual would be the main locus of information, an alternative approach could be to use the communication and reliance popularity scores to determine which team member the MM responses should be compared with to calculate SA. In our sample, such an approach would have led to SA still being based on the Chief of Staff responses in most teams, but based on the Line Leader for two of the teams.

SNA was measured at a single time point (at the end of each training session). It is possible that measuring social dynamics across time-points could provide richer and more detailed data, revealing variability in how information and resources are distributed between team members throughout a developing scenario. As more questions at freeze points may compromise the ecological validity of the approach, researchers should balance the intrusiveness against the need for detailed data. Richer data may also be obtained by using the current approach in longitudinal designs across longer timescales. Trajectories of network properties could then be monitored and evaluated across training programs or interventions for EMTs.

Finally, including objective measures of networks could be a valuable addition to the current approach. For instance, measuring team members' physical proximity to each other, or the frequency and content of communication may complement retrospective self-report data. Similarly, the current approach may be used with objective measures of team performance, such as speed for solving tasks, or the quality of decisions made during training scenarios.

4.5.2: Practical implications. The current study describes the development of standardized tools for measuring and calculating SA and SMM that can be applied by practitioners in the field. The approach is well suited for ecological studies with retrospective self-report measures and could be interpreted by practitioners after regular training exercises. Results indicate that in settings like the one studied here, the organization should strive for a distributed, interaction structure rather than a strongly regularized and hierarchical structure. This could lead practitioners to focus on the team 
interaction in their training, and to implement interventions aimed at improving the interaction. The approach could be used to understand social dynamics differences between teams in an organization.

4.5.3: Conclusion. The current study applied SNA to a selfreport field dataset and found effects of the degree of centralization of EMTs networks on MMs, but no effect of individual network position. This indicates that teams' internal network dynamics are an important source of information, while individual team members' position may be less important. This should be viewed as a promising novel approach that could also be applied in other similar settings.

\section{Author Contributions and Acknowledgements}

Srtrevik conceived of the idea, constructed the materials, collected the data, and developed the shared beliefs measurement. Kvamme and Adrian Renke Bracht wrote their master's thesis based on the current analysis (http://bora.uib.no/handle/1956/17495). Both authors and Bracht collaborated on the pre-registration and developed the social network analysis approach. Both authors contributed in writing the current article.

We appreciate the assistance of Lars Brekke, Tom Pettersen and others in the Equinor second line incident management organization during data collection in 2011. Evelyn-Rose Saus, Sigurd William Hystad, Gry D. Tyssebotn, Annette Marie Kollenborg, Astrid Lovise Westvik and Daniel Hosøy assisted in the data collection. We also appreciate the assistance from Sascha Wolter for mathematical aid on developing alternative measures for network description. This research was supported by the Norwegian Research Council's PETROMAKS Program (grant number 189521).

The current dataset has previously been used for publication. One article described the experiment procedure and shared belief measurement in more detail (Sætrevik \& Eid, 2014), and another described associations that shared beliefs had with between task, team membership, psychophysiology and subjective assessment and situation awareness (Sætrevik, 2015). The dataset has not previously been used in testing of the current hypotheses or for calculating social network measurements.

\section{References}

Balkundi, P., Barsness, Z., \& Michael, J. H. (2009). Unlocking the influence of leadership network structures on team conflict and viability. Small group research, 40(3), 301-322.

Balkundi, P., \& Harrison, D. A. (2006). Ties, leaders, and time in teams: Strong inference about network structure's effects on team viability and performance. Academy of Management Journal, 49(1), 49-68.

Bolstad, C. A., \& Endsley, M. R. (1999). Shared mental models and shared displays: An empirical evaluation of team performance. Paper presented at the Proceedings of the human factors and ergonomics society annual meeting.

Bolstad, C. A., \& Endsley, M. R. (2005). Choosing team collaboration tools: Lessons from disaster recovery efforts. Ergonomics in Design, 13(4), 7-14.

Borgatti, S. P., \& Foster, P. C. (2003). The network paradigm in organizational research: A review and typology. Journal of Management, 29(6), 991-1013.

Borgatti, S. P., Mehra, A., Brass, D. J., \& Labianca, G. (2009). Network analysis in the social sciences. Science, 323(5916), 892-895.

Burt, R. S., Kilduff, M., \& Tasselli, S. (2013). Social network analysis: Foundations and frontiers on advantage. Annu Rev Psychol, 64, 527547.

Cannon-Bowers, J. A., Salas, E., \& Converse, S. (1993). Shared mental models. In J. N. Castellan (Ed.), Individual and group decision making (pp. 221-246). Hillsdale, NJ: Erlbaum.

Carayon, P. (2006). Human factors of complex sociotechnical systems. Applied Ergonomics, 37(4), 525-535. doi:10.1016/j.apergo.2006.04.011

Costa, A. C., Fulmer, C. A., \& Anderson, N. R. (2018). Trust in work teams: An integrative review, multilevel model, and future directions. Journal of Organizational Behavior, 39(2), 169-184.
Cross, R., Borgatti, S. P., \& Parker, A. (2002). Making invisible work visible: Using social network analysis to support strategic collaboration. California management review, 44(2), 25-46.

Cross, R., Rebele, R., \& Grant, A. (2016). Collaborative overload: too much teamwork exhausts employees and saps productivity. Here's how to avoid it.(Spotlight on the Emotional Organization). Harvard Business Review, 94(1-2), 74.

Csardi, G., \& Nepusz, T. (2006). The igraph software package for complex network research. InterJournal, Complex Systems, 1695(5), $1-9$.

Cummings, J. N., \& Cross, R. (2003). Structural properties of work groups and their consequences for performance. Social networks, 25(3), 197-210.

D’Innocenzo, L., Mathieu, J. E., \& Kukenberger, M. R. (2016). A metaanalysis of different forms of shared leadership-team performance relations. Journal of Management, 42(7), 1964-1991.

De Jong, B. A., Dirks, K. T., \& Gillespie, N. (2016). Trust and team performance: A meta-analysis of main effects, moderators, and covariates. Journal of Applied Psychology, 101(8), 1134.

Endsley, M. R. (1988). Situation awareness global assessment technique (SAGAT). In Proceedings of the Academy of Natural Sciences of Philadelphia, 789-795.

Endsley, M. R. (1995). Toward a Theory of Situation Awareness in Dynamic-Systems. Human Factors, 37(1), 32-64. doi:Doi $10.1518 / 001872095779049543$

Grossman, R., \& Feitosa, J. (2018). Team trust over time: Modeling reciprocal and contextual influences in action teams. Human Resource Management Review, 28(4), 395-410.

Hanneman, R. A., \& Riddle, M. (2005). Introduction to social network methods. CA, University of California: Riverside.

Haythornthwaite, C. (1996). Social network analysis: An approach and technique for the study of information exchange. Library \& information science research, 18(4), 323-342.

Henttonen, K. (2010). Exploring social networks on the team level-A review of the empirical literature. Journal of Engineering and Technology Management, 27(1-2), 74-109.

Kilduff, M., \& Tsai, W. (2003). Social networks and organizations: Sage.

Leavitt, H. J. (1951). Some effects of certain communication patterns on group performance. Journal of abnormal psychology, 46(1), 38.

Leenders, R. T. A. J., van Engelen, J. M. L., \& Kratzer, J. (2003). Virtuality, communication, and new product team creativity: a social network perspective. Journal of Engineering and Technology Management, 20(1), 69-92. doi:10.1016/S0923-4748(03)00005-5

Lewis, K., \& Herndon, B. (2011). Transactive memory systems: Current issues and future research directions. Organization science, 22(5), 1254-1265.

Lin, Z., Yang, H., Arya, B., Huang, Z., \& Li, D. (2005). Structural versus individual perspectives on the dynamics of group performance: Theoretical exploration and empirical investigation. Journal of Management, 31(3), 354-380.

Mathieu, J. E., Heffner, T. S., Goodwin, G. F., Salas, E., \& CannonBowers, J. A. (2000). The influence of shared mental models on team process and performance. Journal of Applied Psychology, 85(2), 273.

Mehra, A., Dixon, A., Brass, D., \& Robertson, B. (2006). The Social Network Ties of Group Leaders: Implications for Group Performance and Leader Reputation. Organization Science, 17(1), 64-79. doi:10.1287/orsc. 1050.0158

Mohammadfam, I., Bastani, S., Esaghi, M., Golmohamadi, R., \& Saee, A. (2015). Evaluation of coordination of emergency response team through the social network analysis. Case study: oil and gas refinery. Safety and health at work, 6(1), 30-34.

Monge, P. R., \& Contractor, N. S. (2003). Theories of Communication Networks: United States: Oxford University Press.

Newman, M. E. J. (2010). Networks:An Introduction: An Introduction: United Kingdom: Oxford University Press - Special.

Opsahl, T., Agneessens, F., \& Skvoretz, J. (2010). Node centrality in weighted networks: Generalizing degree and shortest paths. Social Networks, 32(3), 245-251. doi:10.1016/j.socnet.2010.03.006

Osman, M. (2010). Controlling Uncertainty: A Review of Human Behavior in Complex Dynamic Environments. Psychological Bulletin, 136(1), 65-86. doi:10.1037/a0017815 
Rouse, W. B., Cannon-Bowers, J. A., \& Salas, E. (1992). The role of mental models in team performance in complex systems. IEEE transactions on systems, man, and cybernetics, 22(6), 1296-1308.

RStudio Team. (2018). RStudio: Integrated Development for R: RStudio, Inc., Boston, MA. Retrieved from http://www.rstudio.com/.

Salas, E., Sims, D. E., \& Burke, C. S. (2005). Is there a "big five" in teamwork? Small group research, 36(5), 555-599.

Santos, C. M., Uitdewilligen, S., \& Passos, A. M. (2015). A temporal common ground for learning: The moderating effect of shared mental models on the relation between team learning behaviours and performance improvement. European Journal of Work and Organizational Psychology, 24(5), 710-725.

Sarter, N. B., \& Woods, D. D. (1991). Situation awareness: A critical but ill-defined phenomenon. The International Journal of Aviation Psychology, 1(1), 45-57.

Shaw, M. E. (1954). Some effects of unequal distribution of information upon group performance in various communication nets. Journal of Abnormal and Social Psychology, 49(4), 547.

Shaw, M. E. (1964). Communication networks. Advances in experimental social psychology, 1, 111-147.

Sparrowe, R. T., Liden, R. C., \& Kraimer, M. L. (2001). Social Networks and the Performance of Individuals and Groups.(Statistical Data Included). Academy of Management Journal, 44(2), 316. doi: $10.2307 / 3069458$

Stanton, N., Salmon, P., Walker, G., Salas, E., \& Hancock, P. (2017). State-of-science: situation awareness in individuals, teams and systems. Ergonomics, 60(4), 449-466.

Stout, R. J., Cannon-Bowers, J. A., Salas, E., \& Milanovich, D. M. (1999). Planning, shared mental models, and coordinated

\footnotetext{
i The preregistration's description of hypotheses was in some cases ambiguous as to whether the independent variables should be individual or a team level indices of social networks. Since the dependent variable (SMM and SA measures) were for the individual level, it makes sense to also keep the independent variable on individual level, to avoid discarding much of the collected data. Reducing the data to nine datapoints would also make it impossible to draw any conclusions from the analyses. Raw and transformed dataset, and analyses are available in the OSF folder.
}

performance: An empirical link is established. Human Factors, 41(1), 61-71. Retrieved from <Go to ISI $>$ ://000080273600006

Streeter, C. L., \& Gillespie, D. F. (1993). Social Network Analysis. Journal of Social Service Research, 16(1-2), 201-222. doi:10.1300/J079v16n01_10

Sætrevik, B. (2012). A controlled field study of situation awareness and heart rate variability in emergency handling teams. Human Factors: The Journal of the Human Factors and Ergonomics Society, 56(1), 2006-2010. doi:10.1177/1071181312561419

Sætrevik, B. (2015). Psychophysiology, task complexity, and team factors determine emergency response teams' shared beliefs. Safety Science. doi:10.1016/j.ssci.2015.04.017

Sætrevik, B., \& Eid, J. (2014). The 'similarity index' as an indicator of shared mental models and situation awareness in field studies. Journal of Cognitive Engineering and Decision Making, 8(2), 119 136.

Wise, S. (2014). Can a team have too much cohesion? The dark side to network density. European Management Journal, 32(5), 703-711.

Wölfer, R., Faber, N. S., Hewstone, M., \& Marcus, D. K. (2015). Social Network Analysis in the Science of Groups: Cross-Sectional and Longitudinal Applications for Studying Intra- and Intergroup Behavior. Group Dynamics: Theory, Research, and Practice, 19(1), 45-61. doi:10.1037/gdn0000021

Zhang, Z.-X., Hempel, P. S., Han, Y.-L., \& Tjosvold, D. (2007). Transactive memory system links work team characteristics and performance. Journal of Applied Psychology, 92(6), 1722.

\footnotetext{
ii This approach is functionally equivalent to the combination of individual scores into a team's centralization score that was described in the preregistration (see description on the OSF project folder), but we find the current calculation to be more intuitive and algorithmically transparent.
} 